\title{
A microfluidic system for analysis of electrochemical processing using a highly sensitive optical fiber microcavity
}

\author{
Andrzej Krześniak ${ }^{1, \uparrow}$, Tomasz Gabler 2, , Monika Janik ${ }^{2,3}$, Marcin Koba²,4, Martin Jönsson- \\ Niedziółka ${ }^{1, *}$, and Mateusz Śmietana ${ }^{2, *}$ \\ ${ }^{1}$ Institute of Physical Chemistry, Polish Academy of Sciences, \\ Kasprzaka 44/52, 01-224 Warsaw, Poland \\ 2 Warsaw University of Technology, Institute of Microelectronics and Optoelectronics, \\ Warsaw, Koszykowa, 75, Poland \\ 3 Gdańsk University of Technology, Department of Metrology and Optoelectronics, \\ Gabriela Narutowicza 11/12, 80-233 Gdańsk, Poland \\ ${ }_{4}$ National Institute of Telecommunications, Szachowa 1, Warsaw, 04-894, Poland \\ *Corresponding authors: M.Smietana@elka.pw.edu.pl, +48222346364 \\ martinj@ichf.edu.pl, +48223433306 \\ TAuthors contributed equally
}

Microfluidics provide unique possibilities to control tiny volumes of liquids and their composition. To effectively benefit from the advantages of microfluidic solutions they need to be supported by interrogation subsystems, at best also matching the miniature scale. In this work we combined with a microfluidic system a Microcavity in-line Mach-Zehnder Interferometer ( $\mu$ IMZI) induced in the side surface of a single-mode optical fiber using a femtosecond laser micromachining. The $\mu$ IMZI shows capability for investigating optical properties of as small as picoliter volumes with an exceptionally high sensitivity. Here we report numerical analysis and experimental results that show that when the $\mu I M Z I$ is incorporated with the microfluidic system the measurements can be performed with sensitivity exceeding $14,000 \mathrm{~nm} / \mathrm{RIU}$ which is similar to measurements done under static conditions. In a flow injection system we show that a certain amount of liquid and flow rate are required to effectively exchange the liquid in the microcavity, while orientation of the cavity versus the flow direction has a minor impact on the exchange. Finally, we have supported the system by band electrodes making it possible to induce redox reactions in the microchannel and optical detection of flowing products of the reactions. It has been found that thanks to the high sensitivity of the $\mu$ IMZI the products of the reactions can be clearly detected both electrochemically and optically even when the only part of the flowing redox probe is oxidized at the band electrode. This work proves that the proposed solution may offer highly sensitive optical measurements, even when the chemical reactions are not effective in the whole volume of the system.

\section{Introduction}

Microfluidics is a multidisciplinary field of studies aiming to manipulate fluids that are geometrically constrained to a small scale at which surface forces dominate over volumetric forces. Research on microfluidics has started in the 1980 os and since then it has been widely developed in various branches of science[1]. Small size is a defining feature of microfluidic devices, but there are surely other valuable advantages of moving from macro to microscale. Tiny instantaneous volumes (micro to picoliters) associated with microfluidics determine that mass transport is dominated by diffusion [2,3]. Because of that, fluid mixing occurs in a controllable fashion and laminar flow regimes are fully developed. The laminar nature of flow in turn ensures that particle or fluid motions are well-defined and thus they can be engineered towards a particular purpose. For example, 
the flow may be designed to direct and based on their molecular structure distribute a stream of molecules to specific streamlines, e.g., surface-specific antigens, or depending on their physical properties (size) or electrical properties[2].

On top of the laminar flow, large surface-area-to-volume ratios of the fluids ensure thermal homogeneity across the system and rapid heat transfer between the device and the fluid. This advantage ensures uniform reaction conditions, that are particularly important in chemical and biological systems[4]. For these reasons, scaling such systems down to nanoliter or picoliter volumes can fundamentally improve the sensitivity of conducted analysis, simultaneously limit the reagent volume, and therefore reduce final cost of the analysis.

To develop an operational microfluidic-based sensing system, the microfluidic technology needs to be integrated with, e.g., electrical or optical devices, that are required for interrogating of the obtained outcomes. Although the microfluidic systems themselves are usually highly miniaturized, most of them still require support of external, bulky systems, such as e.g., a fluorescent microscope[5,6]. Therefore, a step ahead is to develop miniature interrogation subsystems, but still highly functional and compatible with microfluidics. To improve the sensing efficiency and tackle such challenges as portability and miniaturization of the whole system, optofluidic devices have been proposed where microfluidic (or nanofluidic) systems are integrated with optical sensing devices[7-10]. Such solutions provide conditions for direct interaction between the examined fluid and light, what is expected e.g., in the field of biochemical sensing[11]. Up to date a great variety of planar optical sensing solutions have been combined with microfluidics[11,12]. Nevertheless, the sensitivity of such systems to changes in optical properties of the fluids, such as their refractive index (RI), is often relatively low, i.e., when a spectral shift of a pattern is concerned, it reaches a level of tens up to hundreds of nm per RI unit (RIU). Due to the low sensitivity, such an optical device may not be effective in biochemical applications and detection of biological targets. On top of sensitivity, a crucial issue is a proper integration and alignment of the optical components with microfluidic devices.

In parallel to planar optical sensing solutions, also miniaturized, robust, portable, but most importantly highly sensitive approaches based on optical fibers have been proposed [13-16]. Such devices show several notable advantages over the planar ones. First, they are inexpensive thanks to the well-established fiber manufacturing technology. Moreover, they can easily couple light with microfluidic channels due to their similar size. Also, their immunity to electromagnetic interference or possibility of remote measurements are worth mentioning. In general, optical fibers may be integrated with microfluidics in three different ways. First, thanks to the microstructure of some fibers, they can serve as both light transmission medium and microfluidic channel, as it is in the case of, e.g., a hollow core fiber $[17,18]$. Second, by introduction of the microstructuring processes such as those based on femtosecond (fs) laser micromachining or ion beam lithography creating lab-in-fiber systems[19,20]. Third, the most common one, due to the flexibility in application of various types of sensors, they are achieved by the integration of fiber-based sensors with extrinsic microfluidic channels. Interferometers[21], various gratings[22-24], micro/nano fibers $[25,26]$, and nano-coated fibers (based on surface 
plasmon resonance[27] or lossy-mode resonance[28] effects) have been shown following this approach.

However, integration of the optical fiber sensor with microfluidic system plays crucial role during the sensing process. The way of introducing the analyte to the microchannels and efficiency of this process are especially important. Furthermore, the sensitivity of the sensor to external changes needs to be high enough to enable measurements of the analyte present in the microchannel. Volume of the analyte needed for the detection process is also important. It must be noted that some of the sensors, e.g., gratings[29], or lossy-mode resonance [28], due to their size/length and inaccurately defined sensing area need hundreds of microliters of samples per single analysis. Such volumes may be too large in the case of limited in availability biological and chemical materials. Hence, the structures based on in-fiber microcavities deserve particular attention. Microcavity inline Mach-Zehnder Interferometer ( $\mu \mathrm{IMZI}$ ) is an open cavity micromachined in a fiber cladding and partly in a fiber core. In this configuration, the microcavity splits incoming light into two parts, where one remains in the fiber core (reference beam), while the other one propagates through the cavity (sensing beam). The two beams interfere at the far sidewall of the cavity[30]. Due to the precise, one-step fabrication process, the platform is highly reproducible and provides a well-defined sensing area. The $\mu$ IMZI with a diameter of tens of micrometers allows investigating sub-nanoliter volumes of liquids. The sensitivity of the $\mu$ IMZI reach even over $20,000 \mathrm{~nm} / \mathrm{RIU}[31]$ what makes it a perfect choice for bio- and chemo- sensing, especially when small volumes of analytes are considered. Although the $\mu$ IMZI has been reported for different applications, such as bacteria detection[32,33] and real-time monitoring of isothermal DNA amplification[34], its integration with the extrinsic microfluidic channel has never been reported. Incorporation of the $\mu$ IMZI in a microfluidic system may also be advantageous because it limits evaporation of the analyte and allows a more accurate way of handling a solution inside the microcavity as it can be easily exchanged. Moreover, due to limited exposure of the sensor to changes in environmental conditions, such as drying during the procedure, can be mitigated some additional effects possibly modifying the sensor surface and disturbing the measurements[35].

In this work, we integrate $\mu$ IMZI sensor as an element of a microfluidic system. In contrast to the work of $\mathrm{Li}$ et al. in[36], where the microfluidic channel was laser micromachined together with the microcavity inside an optical fiber, we have implemented an external microfluidic system capable to accommodate also additional elements of the microsystem, such as electrodes. Since the size of the sensing area is a way smaller than for other investigated up to date and integrated with microfluidics optical fiber sensors, the liquid exchange in the cavity has been discussed theoretically and experimentally. Moreover, to show possibility of combining other elements in such an approach, a multi-domain i.e., optical, and electrochemical (EC), $\mu$ IMZI-based microfluidic system has been developed and analysed. In contrast to the sensing concept reported in [37], where EC reactions where initiated directly in the cavity, we investigated the capability of the system for optical measurements of EC reactions taking place outside the cavity. This solution is universal and allows for combining other devices with the system. 


\section{Experimental details}

\section{Fabrication and optical measurements of the microcavities}

Circular in shape microcavities $(54 \mu \mathrm{m}$ diameter, $62.5 \mu \mathrm{m}$ height) were fabricated in side surface of standard Corning $\mathrm{SMF}_{2} 8$ fibers. The micromachining process was performed using an NKT Origami 10XP laser operating at wavelength of $1030 \mathrm{~nm}$. The fiber was irradiated by ca. 400 fs pulses. Fused silica glass shows very low absorption at $1030 \mathrm{~nm}$, and therefore linear absorption of the laser radiation does not occur when the glass is irradiated by the laser beam[38]. The system worked with a repetition rate of 15 $\mathrm{kHz}$. The laser beam was focused on the sample with a suitably designed micromachining setup based on the Newport mFab system. It was equipped with a $20 x$ lens $(\mathrm{NA}=0.30$ ). The laser pulse energy was equal to 6 nJ. Fiber transmission was monitored during the process with an NKT Photonics SuperK COMPACT supercontinuum white light source and a Yokogawa AQ6370D optical spectrum analyzer. The fabrication process was controlled with an in-house developed software.

The spectral responses of the $\mu$ IMZI during its initial testing were measured in possibly wide wavelength range, i.e., from 1100 to $1700 \mathrm{~nm}$, using Yokogawa AQ6370B optical spectrum analyzer and Leukos $\mathrm{SM}_{3} 0$ supercontinuum white light laser source. The RI sensitivity was measured by immersing the sensors in glycerine/water solutions with RI in the range $n_{D}=1.33300-1.40100$ RIU. The $n_{D}$ of the solutions was determined using Rudolph $\mathrm{J}_{57}$ automatic refractometer. The transmission measurements were conducted at constant mechanical tension and temperature $\left(25^{\circ} \mathrm{C}\right)$.

\section{Fabrication of microfluidic system}

For fabrication of microfluidic system glass slides (50 x $25 \times 1.1 \mathrm{~mm}$ ) coated with indium tin oxide (ITO) from Delta Technologies, Ltd. were used as a substrate. The electrodes were made by etching in a solution of $\mathrm{FeCl}_{3}$ as described previously in [3]. First, shape of the electrodes was defined by a kapton tape cut using GCC LaserPro C180II Laser Engraver controlled by a computer program. The tape was used to mask the ITO electrodes during the etching process. The width of the working (WE) and counter electrode (CE) was $8 \mathrm{~mm}$ and $10 \mathrm{~mm}$, respectively. Next, the CE was covered with gold using a sputtering deposition method to increase its electrochemical activity. A $5 \mathrm{~nm}$ layer of Ti was used under the gold film to promote adhesion.

Next, for microchannel fabrication a PDMS (Sylgard 184 Silicone, a two-part poly (dimethylsiloxane) elastomer from Dow Corning) was used. Firstly, a matrix for the channel was designed and printed in polylactic acid (PLA) using ${ }_{3} \mathrm{D}$ printer Ultimaker ${ }_{2}+$. Then, a microchannel was made by pouring the monomers of PDMS into the matrix and drying for 90 minutes at $65^{\circ} \mathrm{C}$. As a result, a $500 \mu \mathrm{m}$ wide, $180 \mu \mathrm{m}$ high, and $2 \mathrm{~cm}$ long microchannel was received. Holes for the liquid inlet and outlet, as well as the reference electrode were made in the PDMS. Finally, an optical fiber with the microcavity was installed between WE and CE and the PDMS as shown in Fig. 1.

\section{Flow injection and electrochemical experiment}

The EC response of the sensor has been studied using a portable PalmSens4 potentiostat controlled to a computer equipped with PSTrace 5.6 software. Bare ITO electrode was used as WE, whereas ITO coated with gold served as CE. An external Ag/ AgCl electrode 


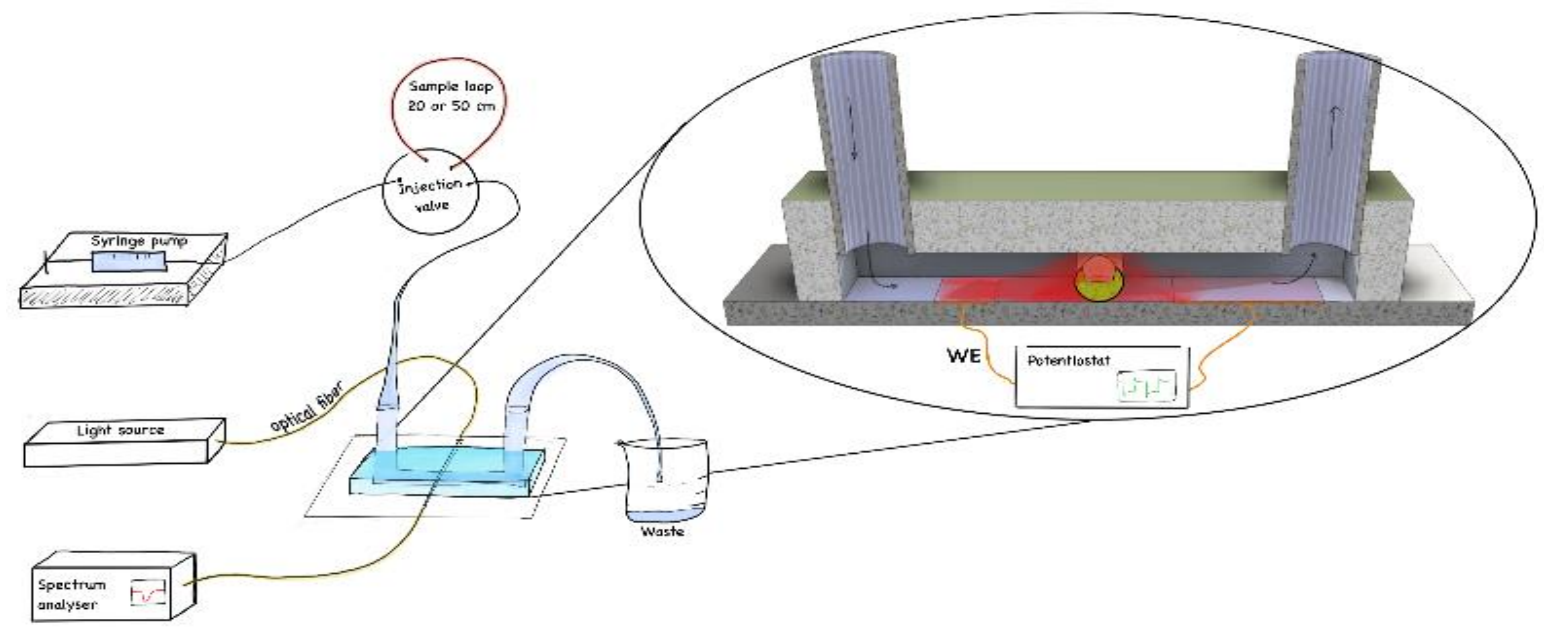

Fig. 1 Schematic representation of the experimental setup

was used as a RE. Copper tape was used to provide an electric contact between ITO electrodes and the potentiostat. For experiments we used following solutions: 1 mM 1,1'ferrocenedimethanol $(\mathrm{FCDM})$ in $0.1 \mathrm{M} \mathrm{KCl}, 10 \mathrm{mM} \mathrm{K}_{3} \mathrm{Fe}(\mathrm{CN})_{6}$ in $0.1 \mathrm{M} \mathrm{KCl}$ and $10 \mathrm{mM}$ $\mathrm{K}_{4} \mathrm{Fe}(\mathrm{CN})_{6}$ in $0.1 \mathrm{M} \mathrm{KCl}$. For the preparation of solutions, we used: $\mathrm{KCl}$ powder from POCh (pure p.a.), 98\% FcDM from ACROS Organics, $\mathrm{K}_{3} \mathrm{Fe}(\mathrm{CN})_{6}$ powder from SigmaAldrich, $\mathrm{K}_{4} \mathrm{Fe}(\mathrm{CN})_{6}{ }_{3} \mathrm{H}_{2} \mathrm{O}$ powder from $\mathrm{POCh}$ and deionized water from Millipore system. For determining the flow rate, a Harvard Apparatus Syringe infusion pump 11 equipped with silicone tubing (inner diam. $0.8 \mu \mathrm{m}$ ) was used to pump solutions into the microchannel. For the flow injection experiments an injection valve (Cheminert, Vici) with two different of sample loops were used, i.e., $20 \mathrm{~cm}$ and $50 \mathrm{~cm}$ long. These have an injection volume of $100 \mu \mathrm{l}$ and $250 \mu \mathrm{l}$, respectively. The flow rate was set to $100 \mu \mathrm{l} / \mathrm{min}$, which is the highest value that ensures that the concentration in the microchannel reaches the same value as in the sample loop (Fig $\mathrm{S}_{2}$ ). For EC measurements the flow rate was $5-10 \mu \mathrm{l} / \mathrm{min}$. The combined EC-optical setup used for measurements is shown in Fig. 1.

\section{Numerical analysis}

Numerical analysis of the flow was performed using the Comsol 5.6 software with the laminar flow module. The EC response was modelled using the electrochemistry module (see Supplementary Information for details), where the electrode reaction was modelled using the Butler-Volmer equation with the exchange current density following the mass action law[39]. The rectangular channel, cavity and electrode geometries were described in $3 \mathrm{D}$ coordinates and the change in concentration of the oxidized form of the redox probe during the experiment was monitored as the average at the bottom of the cavity. The Taylor dispersion in the injection loop was simulated in a $2 \mathrm{D}$ cylindrically symmetrical model with a $10 \mathrm{~cm}$ connection tube after the loop. The output concentration from the connection tube was used as input into the microchannel. The angle of the cavity in the flow channel was varied to see the effect on the response time. To investigate the response of the cavity to EC reaction in the channel, we simulated the oxidation of a generic redox probe at an $8 \mathrm{~mm}$ wide electrode $1 \mathrm{~mm}$ upstream from the fiber. As in the previous case, the concentration of the oxidized form at the bottom of 
(A)

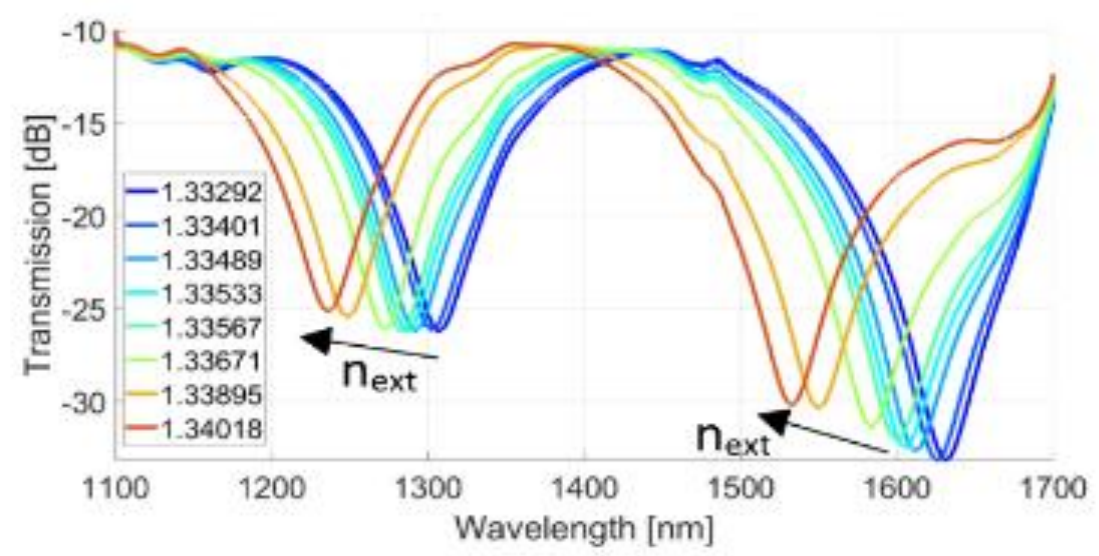

(B)

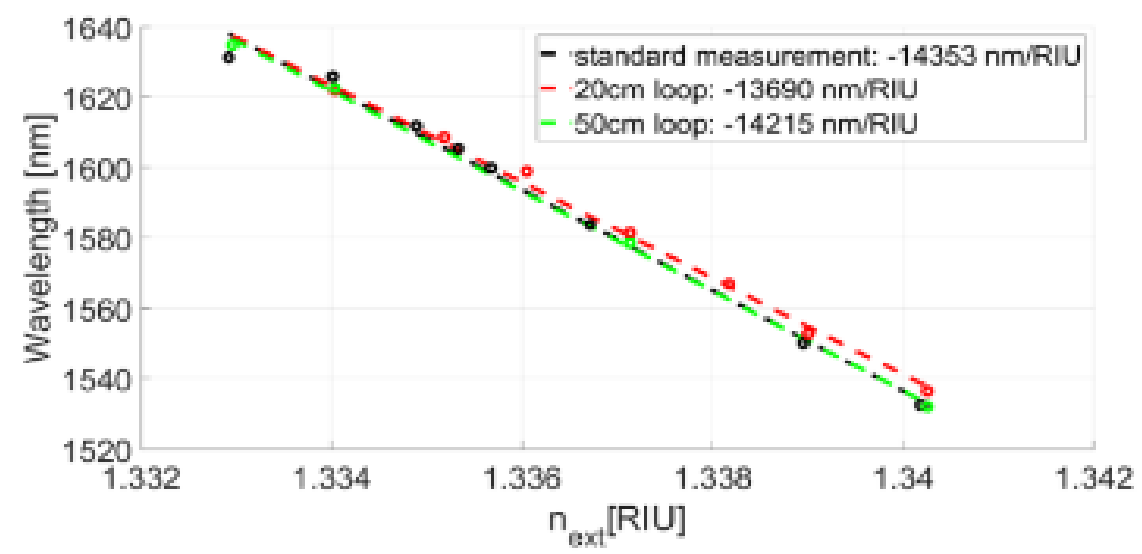

Fig. 2 (A) Transmission spectra of $\mu$ IMZI filled with next ranging from 1.33292 to 1.34018 RIU and (B) shows corresponding minimum wavelength plotted vs next in three differen1 measurement setups: static (standard) measurement, and dynamic measurements using $20 \mathrm{~cm}$ and $50 \mathrm{~cm}$ long loop.

the cavity was monitored as a proxy for the optical response. The EC reaction was simulated chronoamperometrically as a potential step to large overpotential and a reverse step after $15 \mathrm{~s}$. The simulation was performed for flowrates from 2 to $25 \mu \mathrm{min}^{-1}$ for the electrochemical system and $25-250 \mu \mathrm{min}^{-1}$ for the flow injection system.

\section{Results and discussion}

\section{RI sensing}

The effect of changes of RI in the microcavity for standard conditions, i.e., the liquid is exchanged manually using a pipette, is shown in Fig. 2(A). When the RI increases the minima shift towards shorter wavelength and reduce their transmission. The phenomenon is originated by change in guiding conditions of the mode propagating in the cavity and interference at the distant cavity wall [34]. The RI sensitivity of the fabricated $\mu$ IMZI measured in standard and microfluidic conditions has been compared in Fig. 2(B). It can be seen that for the longer loop applied, i.e., longer exposure to the elevated $n_{\text {ext }}$, the performance of the device tends to the one investigated at standard conditions, while for the shorter loop, it is slightly lower. The results indicate that 


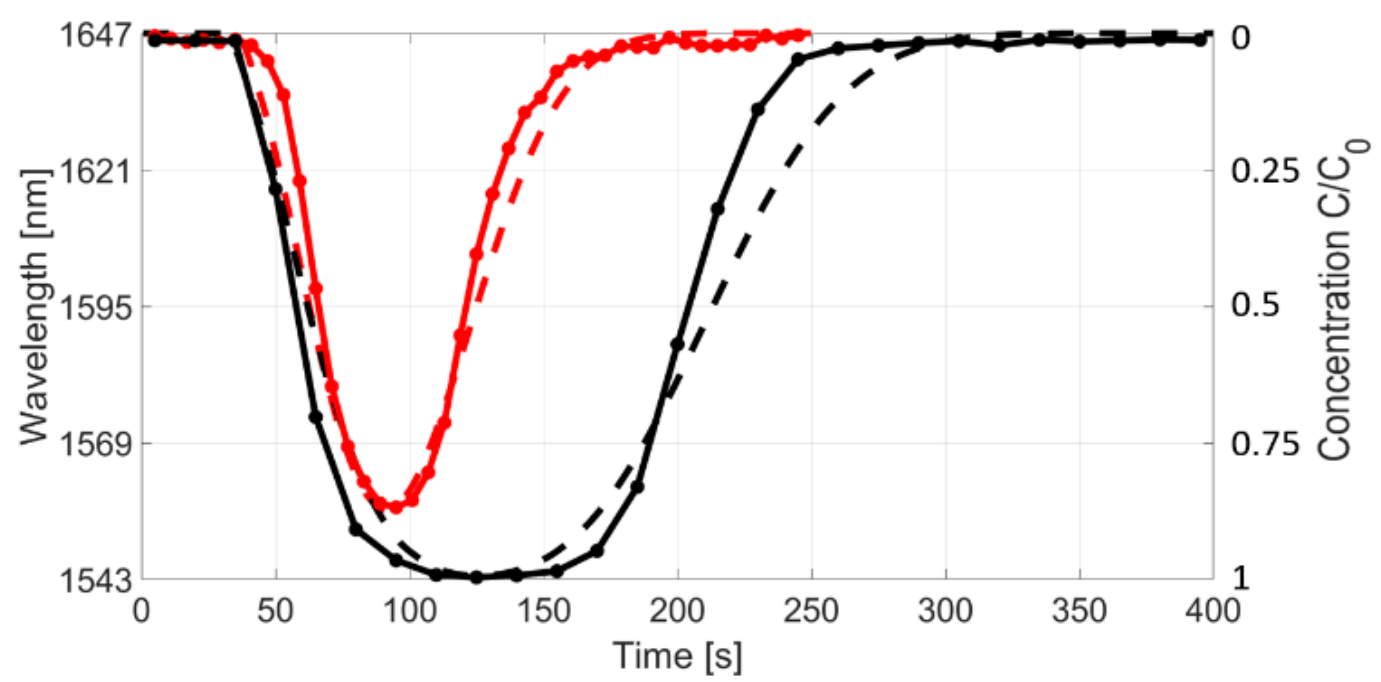

Fig. 3 Concentration profiles, comparing the $20 \mathrm{~cm}$ loop (red lines) and the $50 \mathrm{~cm}$ loop (black lines). Numerical results are marked with dash lines, experimental results are marked with solid lines. In both cases the next change was in range 1.33296 to 1.34025 RIU. The simulations and the experiments were done for flow rate $100 \mu \mathrm{l} / \mathrm{min}$.

exchange of the liquid in the microcavity at the microfluidic conditions requires certain amount of liquid to effectively exchange.

The impact of the exposure of the cavity to liquid of the different $n_{\text {ext }}$, where in this experiment exposure has been set by the length of the loop, is shown in Fig. 3 . Experimental data and numerical analysis agree well. The RI of the liquid in the loop was 1.34025 RIU while that of the background liquid was 1.33296 RIU. Due to Taylor dispersion in the connection tube, the liquid in the sample loop is slightly diluted and the simulations show that the ultimate concentration (and thus RI) reaches only $85 \%$ of its full value for the smaller volume loop. Taylor dispersion is the process through which the difference in flow velocity along the radial direction of the tube leads to a smearing out of the concentration distribution along the direction of the flow [40]. When the concentration/RI of the liquid coming from the loop increases, the wavelength corresponding to the transmission minimum follows and its value decreases. For shorter loop, in agreement with the simulations the shift in resonance wavelength is only about $85 \%$ of the value for the longer loop.

It was noted, that on top of volume of the exchanged liquid discussed above, also orientation of the cavity versus the flow direction could potentially be a factor influencing the effectiveness of the exchange. Thus, we have performed a set of numerical analysis aiming to identify the impact of the cavity placement in the microfluidic system. The results are shown in Fig. $S_{2}$ and $S_{3}$ of the Supplementary Information and indicate negligible influence of the cavity orientation vs direction of the flow on the exchange of liquid within the timescale of the experiments. The flow rate has a large influence on the final concentration in the channel. For flow rates above 100 $\mu \mathrm{l} / \mathrm{min}$ the ultimate concentration is less than the one in the sample loop (Fig. $\mathrm{S}_{2}$ ), therefore experiments were performed using this flow rate to limit the time needed for the measurements. 
Next, oxidized and reduced forms of a common redox probe in $\mathrm{KCl}$, as well as $\mathrm{KCl}$ as a base electrolyte, were used as liquids flushing the cavity in the microsystem. It can be seen in Fig. 4(A) that when any of the probes is introduced to the supporting electrolyte, the wavelength corresponding to the transmission minimum decreases, but it decreases more (by about $2 \mathrm{~nm}$ ) when $\mathrm{K}_{4} \mathrm{Fe}(\mathrm{CN})_{6}$ is applied. $\mathrm{K}_{4} \mathrm{Fe}(\mathrm{CN})_{6}$ has a higher $\mathrm{RI}$ than the one of $\mathrm{K}_{3} \mathrm{Fe}(\mathrm{CN})_{6}$. A slight increase in the drop with exchange sessions can be seen for

(A) Potential [V]

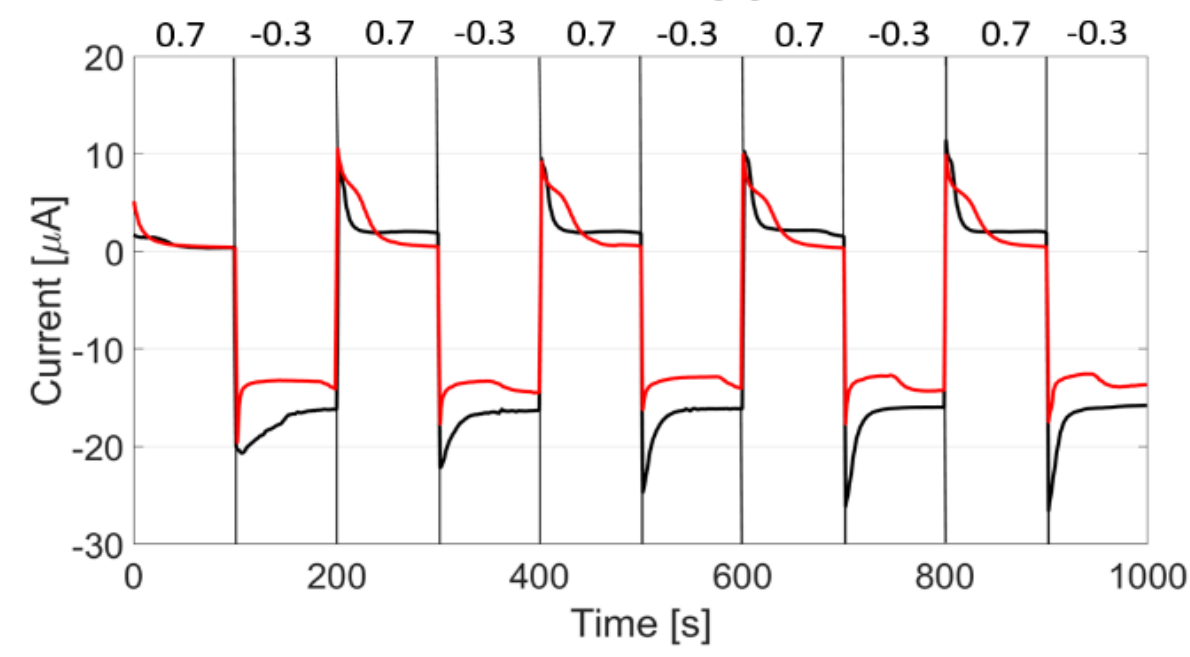

(B)

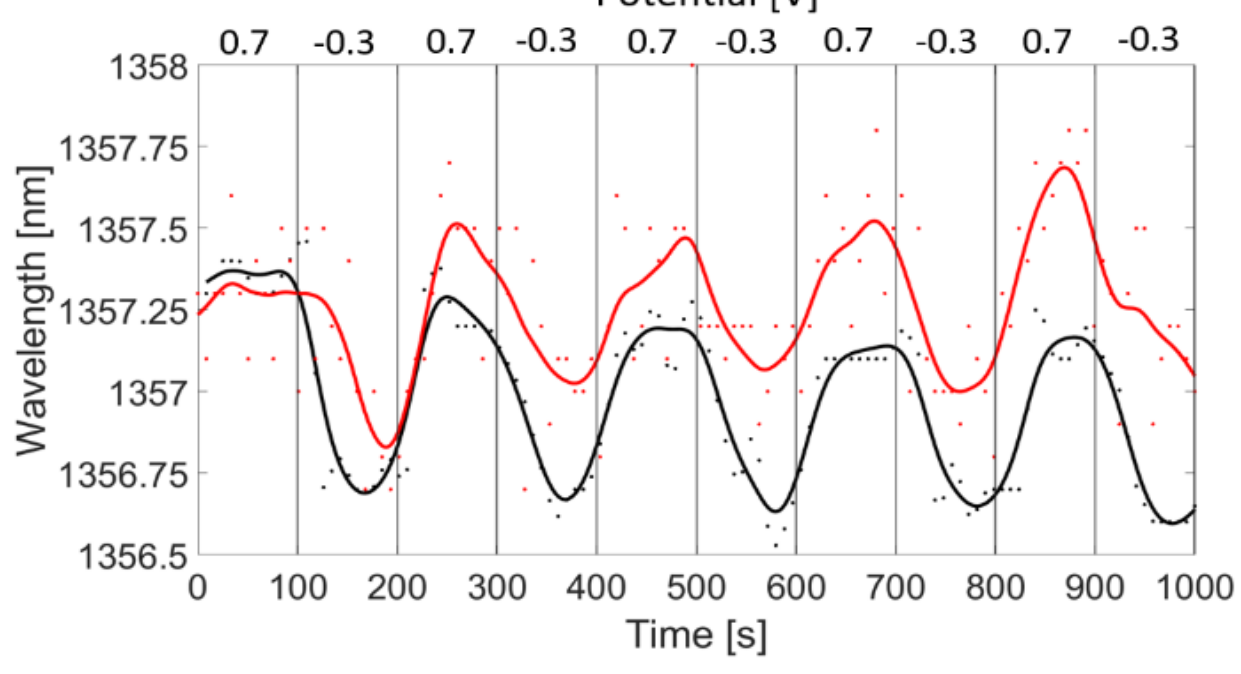

(C)

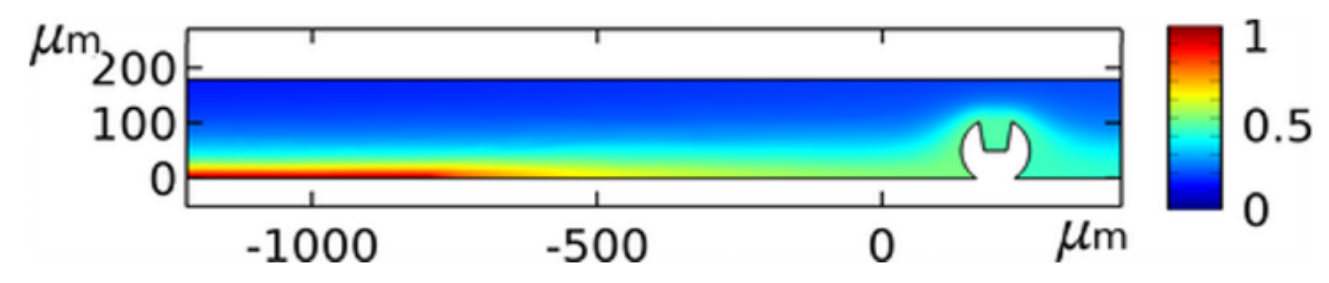

Fig. 5 Current (A) and wavelength (B) shift in time during CA for different $\mathrm{K}_{3} \mathrm{Fe}(\mathrm{CN}) 6$ flow speeds: $10 \mu \mathrm{L} / \mathrm{min}$ and $2 \mu \mathrm{L} / \mathrm{min}$ (black and red solid lines respectively). (C) shows the simulated concentration profile of the product of the electrochemical reaction at the band working electrode at $\mathrm{t}={ }_{15} \mathrm{~s}$ in a cross-section through the center of the channel for $\mathrm{V}_{\mathrm{f}}=\mathbf{1 0} \mu \mathrm{L} / \mathrm{min}$. 
$\mathrm{K}_{4} \mathrm{Fe}(\mathrm{CN})_{6}$. The exchange between the two forms shown in Fig. 4 (B) correspond well with these results and here also wavelength shift reaching about $2 \mathrm{~nm}$ can be noticed.

\section{Monitoring of EC processing}

Following the reported above results, the oxidation and reduction of the redox probe was performed directly in the microfluidic channel using the integrated electrodes and a chronoamperometric configuration of the EC setup. The potential applied to the WE was switched between $-0.3 \mathrm{~V}$ and $+0.7 \mathrm{~V}$ every $100 \mathrm{~s}$. The value $+0.7 \mathrm{~V}$ is well above the oxidation potential of $\mathrm{Fe}(\mathrm{CN}) 6^{4^{-}}$which initializes the oxidation of the redox probe, i.e., conversion of $\mathrm{Fe}(\mathrm{CN}) 6^{4^{-}}$to $\mathrm{Fe}(\mathrm{CN})_{6^{3-}}$. The impact of changes in the applied potential to electrical (Fig. 5(A)) and optical properties (Fig. 5(B)) can be seen at different flow rates. Decrease of $\mathrm{E}$ is followed in by current and wavelength in EC and optical setup, respectively. While the current between the stages differs by over $15 \mu \mathrm{A}$, the wavelength shifts by about $0.7 \mathrm{~nm}$ what is significantly lower than when oxidized and reduced forms of the probe were supplied through the flow injection loop (Fig. 5(B)). This is in relatively good agreement with the simulations that show that for a flow rate of 10 $\mu \mathrm{l} / \mathrm{min}$ the concentration of oxidized form of the redox probe reaches about $45 \%$ of the concentration of the reduced form in the incoming liquid (Fig. $\mathrm{S}_{4}$ ). The reason is that in the EC experiments the oxidized form is created only at the bottom of the channel (Fig. ${ }_{5} \mathrm{C}$ ), while in the flow injection the channel is filled homogeneously with the injected sample. This difference also means that in the EC setup, the response is quite sensitive to the detailed flow of the liquid around the fiber. This can explain why the experimental data correspond less well to the simulation in case of EC actuation as compared to the flow injection. When different flowrates are applied, a clear difference is seen in the current response, but the optical response is quite similar in both cases. In contrast to the simulations, the response at low flowrate is even smaller than for the higher flow. At very low flow rates effects not considered in the simulations, such as convection due to temperature differences in the system, will be more pronounced than when the forced convective flow is higher. This might explain the discrepancy between the simulated and experimental data. 


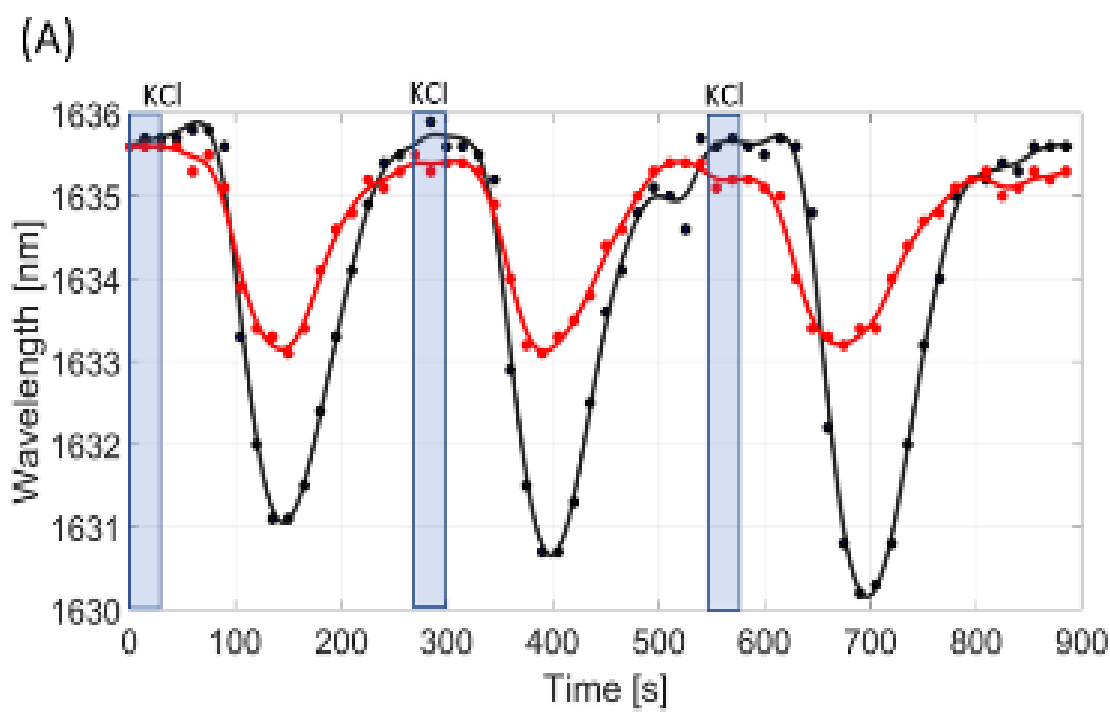

(B)

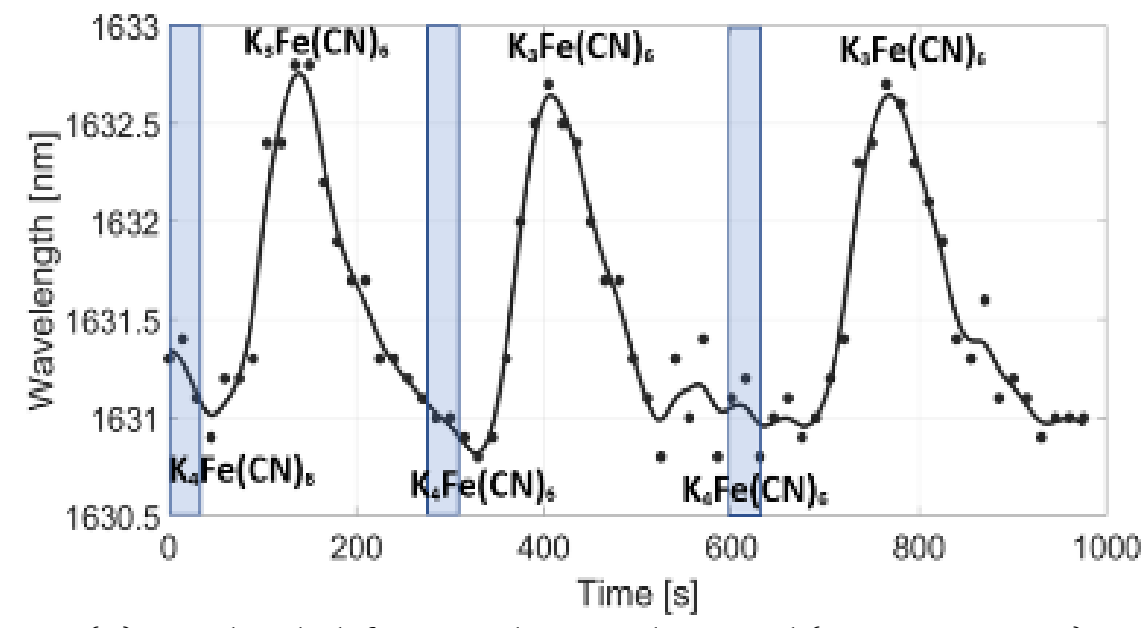

Fig. 4 (A) Wavelength shift in time during exchange $\mathrm{KCl}(\mathrm{nD}=1.33409 \mathrm{RIU})$ to $\mathrm{K}_{4} \mathrm{Fe}(\mathrm{CN}) 6$ $(\mathrm{nD}=1.33437 \mathrm{RIU})$ (black line) and during exchange $\mathrm{KCl}$ to $\mathrm{K}_{3} \mathrm{Fe}(\mathrm{CN}) 6(\mathrm{nD}=1.33424 \mathrm{RIU})$ (red line). (B) Wavelength shift in time during exchange $\mathrm{K}_{4} \mathrm{Fe}(\mathrm{CN}) 6(\mathrm{nD}=1.33437 \mathrm{RIU})$ tc $\mathrm{K}_{3} \mathrm{Fe}(\mathrm{CN}) 6(\mathrm{nD}=1.33424 \mathrm{RIU})$. Both in $(\mathrm{A})$ and $(\mathrm{B})$ the exchange of liquids was repeated three times in $50 \mathrm{~cm}$ loop. Blue areas indicate the approximate moment of switching betweer fluids.

\section{Conclusions}

Highly functional microfluidic systems need to be supported by subsystem making possible to efficiently analyse properties of liquids flowing in the microchannel. In this work we have shown both numerical and experimental analysis of Microcavity in-line Mach-Zehnder Interferometer ( $\mu \mathrm{IMZI}$ ) induced in the side surface of a single-mode optical fiber and a microfluidic system. First, it must be noted that after the integration $\mu$ IMZI maintains its high sensitivity to refractive index changes in the cavity. Second, amount of liquid flowing in the microchannel and the flow rate need to be adjusted to effectively exchange the liquid in the microcavity, but orientation of the cavity versus the flow direction has a minor impact on the exchange. Finally, we additionally integrated electrodes with the system making possible to initiate reduction and oxidation reactions directly in the microchannel. It has been shown that even when the oxidized 
or reduced form is created in part of the channel, the products of the reactions can be identified both electrochemically and optically. The finding indicates that capability to modify the properties of the liquids outside the microcavity and thanks to microfluidic system analyse them in the flow. The results allow for considering the developed system as a promising and universal solution for highly sensitive biochemical analysis in the microscale.

\section{Conflicts of interest}

There are no conflicts to declare.

\section{Acknowledgements}

This work was supported in Poland by the National Science Centre (NCN) under grants No. $\mathrm{NCN} \mathrm{2018/29/B/ST7/O2552.}$

\section{References}

[1] G.M. Whitesides, The origins and the future of microfluidics, Nature. 442 (2006) 368-373. https://doi.org/10.1038/nature05058.

[2] D.T. Chiu, A.J. deMello, D. Di Carlo, P.S. Doyle, C. Hansen, R.M. Maceiczyk, R.C.R. Wootton, Small but Perfectly Formed? Successes, Challenges, and Opportunities for Microfluidics in the Chemical and Biological Sciences, Chem. 2 (2017) 201-223.

https://doi.org/10.1016/j.chempr.2017.01.009.

[3] E. Rozniecka, M. Jonsson-Niedziolka, A. Celebanska, J. Niedziolka-Jonsson, M. Opallo, Selective electrochemical detection of dopamine in a microfluidic channel on carbon nanoparticulate electrodes, Analyst. 139 (2014) 2896. https://doi.org/10.1039/c3ano2207b.

[4] S.H. Huang, Y.S. Chang, J.M.J. Juang, K.W. Chang, M.H. Tsai, T.P. Lu, L.C. Lai, E.Y. Chuang, N.T. Huang, An automated microfluidic DNA microarray platform for genetic variant detection in inherited arrhythmic diseases, Analyst. 143 (2018) 1367-1377.

https://doi.org/10.1039/c7ano1648d.

[5] S.P. Zhao, Y. Ma, Q. Lou, H. Zhu, B. Yang, Q. Fang, Three-Dimensional Cell Culture and Drug Testing in a Microfluidic Sidewall-Attached Droplet Array, Anal. Chem. 89 (2017) 1015310157. https://doi.org/10.1021/acs.analchem.7bo2267.

[6] S. Wang, Z. Ai, Z. Zhang, M. Tang, N. Zhang, F. Liu, G. Han, S.L. Hong, K. Liu, Simultaneous and automated detection of influenza A virus hemagglutinin $\mathrm{H}_{7}$ and $\mathrm{H}_{9}$ based on magnetism and size mediated microfluidic chip, Sensors Actuators, B Chem. 308 (2020) 127675. https://doi.org/10.1016/j.snb.2020.127675.

[7] C. Monat, P. Domachuk, B.J. Eggleton, Integrated optofl uidics : A new river of light, Nat. Photonics. 1 (2007) 106-114. https://doi.org/10.1038/nphoton.2006.96.

[8] D. Ozcelik, H. Cai, K.D. Leake, A.R. Hawkins, H. Schmidt, Optofluidic bioanalysis: Fundamentals and applications, Nanophotonics. 6 (2017) 647-661. https://doi.org/10.1515/nanoph-2016-0156.

[9] J.T. Butement, H.C. Hunt, D.J. Rowe, P. Minzioni, R. Osellame, C. Sada, S. Zhao, Roadmap for optofluidics, J. Opt. 19 (2017) 93003.

[10] C. Song, S.H. Tan, A Perspective on the Rise of Optofluidics and the Future, Micromachines. 8 (2017) 1-17. https://doi.org/10.3390/mi8050152.

[11] K.E. Bates, H. Lu, Biophysical Perspective Optics-Integrated Microfluidic Platforms for 
Biomolecular Analyses, Biophys. J. 110 (2016) 1684-1697.

https://doi.org/10.1016/j.bpj.2016.03.018.

[12] D. Barshilia, L.-K. Chau, G.-E. Chang, Low-cost planar waveguide-based optofluidic sensor for real-time refractive index sensing, Opt. Express. 28 (2020) 27337-27345.

[13] Y. Zhao, X. guang Hu, S. Hu, Y. Peng, Applications of fiber-optic biochemical sensor in microfluidic chips: A review, Biosens. Bioelectron. 166 (2020) 112447.

https://doi.org/10.1016/j.bios.2020.112447.

[14] S. Pissadakis, Lab-in-a-fiber sensors: A review, Microelectron. Eng. 217 (2019) 1-21. https://doi.org/10.1016/j.mee.2019.111105.

[15] R. Blue, A. Duduś, D. Uttamchandani, A Review of Single-Mode Fiber Optofluidics, IEEE J. Sel. Top. Quantum Electron. 22 (2016) 380-391. https://doi.org/10.1109/JSTQE.2015.2466071.

[16] L. Li, Y. nan Zhang, Y. Zhou, W. Zheng, Y. Sun, G. Ma, Y. Zhao, Optical Fiber Optofluidic BioChemical Sensors: A Review, Laser Photonics Rev. 15 (2021) 1-56.

https://doi.org/10.1002/lpor.202000526.

[17] L. Shao, Z. Liu, J. Hu, D. Gunawardena, H.Y. Tam, Optofluidics in microstructured optical fibers, Micromachines. 9 (2018) 1-19. https://doi.org/10.3390/mig040145.

[18] R. Gao, D.F. Lu, M.Y. Zhang, Z.M. Qi, Optofluidic Immunosensor Based on Resonant Wavelength Shift of a Hollow Core Fiber for Ultratrace Detection of Carcinogenic Benzo[a]pyrene, ACS Photonics. 5 (2018) 1273-1280.

https://doi.org/10.1021/acsphotonics.7bo10o9.

[19] K. Sugioka, Y. Cheng, Femtosecond laser processing for optofluidic fabrication, Lab Chip. 12 (2012) 3576-3589. https://doi.org/10.1039/c2lc40366h.

[20] M. Haque, K.K.C. Lee, S. Ho, L.A. Fernandes, P.R. Herman, Chemical-assisted femtosecond laser writing of lab-in-fibers, Lab Chip. 14 (2014) 3817-3829.

https://doi.org/10.1039/c4lcoo648h.

[21] D. Zhang, H. Wei, S. Krishnaswamy, 3D Printing Optofluidic Mach-Zehnder Interferometer on a Fiber Tip for Refractive Index Sensing, IEEE Photonics Technol. Lett. 31 (2019) 1725-1728. https://doi.org/10.1109/LPT.2019.2943897.

[22] S.M. Lee, M.Y. Jeong, S.S. Saini, Etched-core fiber Bragg grating sensors integrated with microfluidic channels, J. Light. Technol. 30 (2012) 1025-1031.

https://doi.org/10.1109/JLT.2011.2167220.

[23] B. Jiang, H. Dai, Y. Zou, X. Chen, Continuous detection of micro-particles by fiber Bragg grating Fabry-Pérot flow cytometer, Opt. Express. 26 (2018) 12579.

https://doi.org/10.1364/oe.26.012579.

[24] M.S. Murib, D. Martens, P. Bienstman, Label-free real-time optical monitoring of DNA hybridization using SiN Mach-Zehnder interferometer-based integrated biosensing platform, J. Biomed. Opt. 23 (2018) 1. https://doi.org/10.1117/1.jbo.23.12.127002.

[25] S.C. Yan, F. Xu, A review on optical microfibers in fluidic applications, J. Micromechanics Microengineering. 27 (2017) 1-20. https://doi.org/10.1088/1361-6439/aa7a45.

[26] L. Liang, C. Zhao, F. Xie, L.-P. Sun, Y. Ran, L. Jin, B.-O. Guan, Sensitivity enhancement of a fiber-based interferometric optofluidic sensor, Opt. Express. 28 (2020) 24408.

https://doi.org/10.1364/oe.400325.

[27] Y. Yang, S. Bandyopadhyay, L. Shao, J. Jiang, Z. Peng, S. Liu, J. Hu, P.P. Shum, J. Hu, X. Zhang, Anomalous Sensitivity Enhancement of D-Shaped Fiber-Based Sandwiched Structure Optofluidic Sensor, IEEE Access. 8 (2020) 105207-105216. https://doi.org/10.1109/ACCESS.2020.2999733.

[28] F. Chiavaioli, P. Zubiate, I. Del Villar, C.R. Zamarrenİo, A. Giannetti, S. Tombelli, C. Trono, F.J. Arregui, I.R. Matias, F. Baldini, Femtomolar Detection by Nanocoated Fiber Label-Free 
Biosensors, ACS Sensors. 3 (2018) 936-943. https://doi.org/10.1021/acssensors.7boog18.

[29] M. Yin, B. Huang, S. Gao, A.P. Zhang, X. Ye, Optical fiber LPG biosensor integrated microfluidic chip for ultrasensitive glucose detection, Biomed. Opt. Express. 7 (2016) 2067. https://doi.org/10.1364/boe.7.002067.

[30] M. Janik, A.K. Myśliwiec, M. Koba, A. Celebańska, W.J. Bock, M. Śmietana, Sensitivity Pattern of Femtosecond Laser Mach-Zehnder Interferometers , as Applied to Small-Scale Refractive Index Sensing, IEEE Sens. J. 17 (2017) 3316-3322.

[31] M. Janik, S. Member, T. Eftimov, M. Koba, M. Smietana, W.J. Bock, L. Member, Tailoring Properties of Microcavity In-Line Mach-Zehnder Interferometer by the Microcavity Enlargement Using Femtosecond Laser, J. Light. Technol. 37 (2019) 4501-4506. https://doi.org/10.1109/JLT.2019.2907661.

[32] M. Janik, E. Brzozowska, P. Czyszczoń, A. Celebańska, M. Koba, A. Gamian, W.J. Bock, M. Śmietana, Optical fiber aptasensor for label-free bacteria detection in small volumes, Sensors Actuators, B Chem. 330 (2021) 1-10. https://doi.org/10.1016/j.snb.2020.129316.

[33] M. Janik, M. Koba, A. Celebańska, W.J. Bock, M. Śmietana, Live E. coli bacteria label-free sensing using a microcavity in-line Mach-Zehnder interferometer, Sci. Rep. 8 (2018) 1-8. https://doi.org/10.1038/s41598-018-35647-2.

[34] M. Janik, S.V. Hamidi, M. Koba, J. Perreault, R. Walsh, W.J. Bock, M. Śmietana, Real-time isothermal DNA amplification monitoring in picoliter volumes using an optical fiber sensor, Lab Chip. 21 (2021) 397-404. https://doi.org/10.1039/DoLCo1069C.

[35] M. Janczuk-Richter, M. Dominik, M. Koba, P. Mikulic, W.J. Bock, S. MacKowski, M. JonssonNiedziolka, J. Niedziolka-Jonsson, M. Smietana, Water-Induced Fused Silica Glass Surface Alterations Monitored Using Long-Period Fiber Gratings, J. Light. Technol. 37 (2019). https://doi.org/10.1109/JLT.2019.2909947.

[36] Z. Li, C. Liao, D. Chen, J. Song, W. Jin, G.-D. Peng, F. Zhu, Y. Wang, J. He, Y. Wang, Label-free detection of bovine serum albumin based on an in-fiber Mach-Zehnder interferometric biosensor, Opt. Express. 25 (2017) 17105. https://doi.org/10.1364/OE.25.017105.

[37] T. Gabler, A. Krześniak, M. Janik, A. Myśliwiec, M. Koba, J. Buczyńska, M. JönssonNiedziółka, M. Śmietana, Electrochemistry in an optical fiber microcavity - optical monitoring of electrochemical processes in picoliter volumes, Lab Chip. 21 (2021) 2763-2770. https://doi.org/10.1039/D1LCo0324K.

[38] J. Qiu, K. Miura, K. Hirao, Femtosecond laser-induced microfeatures in glasses and their applications, J. Non. Cryst. Solids. 354 (2008) 1100-1111.

https://doi.org/10.1016/j.jnoncrysol.2007.02.092.

[39] E.J.F. Dickinson, A.J. Wain, The Butler-Volmer equation in electrochemical theory: Origins, value, and practical application, J. Electroanal. Chem. 872 (2020) 114145. https://doi.org/10.1016/j.jelechem.2020.114145.

[40] G.I. Taylor, Dispersion of soluble matter in solvent flowing slowly through a tube, Proc. R. Soc. London. Ser. A. Math. Phys. Sci. 219 (1953) 186-203. https://doi.org/10.1098/rspa.1953.0139. 